\title{
Measurement of observables sensitive to coherence effects in hadronic $Z$ decays with the OPAL detector at LEP
}

\author{
Nadine Fischer \\ Karlsruher Institut für Technologie, Karlsruhe, Germany \\ now at Monash University, Melbourne, Australia \\ E-mail: Nadine.Fischer@monash.edu \\ Stefan Gieseke \\ Karlsruher Institut für Technologie, Karlsruhe, Germany \\ E-mail: stefan.giesekeakit.edu
}

\section{Stefan Kluth}

Max-Planck-Institut für Physik, München, Germany

E-mail: stefan.kluth@mpp.mpg . de

\section{Simon Plätzer}

DESY, Hamburg, Germany

now at Durham University, Durham, UK

E-mail: simon.platzer@durham.ac.uk

\section{Peter Skands}

CERN, Geneve, Switzerland

now at Monash University, Melbourne, Australia

E-mail: peter.skands@monash. edu

\section{Andrii Verbytskyi*}

Max-Planck-Institut für Physik, München, Germany

E-mail: andrii.verbytskyi@mpp.mpg . de

A study of QCD coherence is presented based on a sample of about $397000 e^{+} e^{-}$hadronic annihilation events collected at $\sqrt{s}=91 \mathrm{GeV}$ with the OPAL detector at LEP. The study is based on four recently proposed observables that are sensitive to coherence effects in the perturbative regime. The measurement of these observables is presented, along with a comparison with the predictions of different parton shower models. The models include both conventional parton shower models and dipole antenna models. Different ordering variables are used to investigate their influence on the predictions.

The European Physical Society Conference on High Energy Physics 22-29 July 2015

Vienna, Austria

\footnotetext{
*Speaker.
} 


\section{Introduction}

The quantum chromodynamics (QCD) is crucially important for the description of the interactions of high energy particles. Therefore, for the deeper understanding of these it is important to account for the QCD effects and to model them as accurately as possible. Recent progress in the theory allows to model QCD effects with much higher precision than in the past. Therefore, now it is possible to study the effects that previously were known only qualitatively. In this case it is important to admit that not only the data from the contemporary experiments, but also the results from previous measurements can be used for the studies.

The subject of the presented analysis is the study of destructive interference effect between colour-connected partons, colour coherence, and tests of different theoretical approaches for the modelling of the latter in the modern Monte Carlo (MC) event generators. The tested theoretical models are based on the QCD parton shower approximation with differences in the choice of the evolution/ordering variables. The validation and fine tuning of these models and can help to better describe the experimental results for which the QCD effects play an important role. For instance, it may help to constrain the searches of physics beyond the Standard Model at the LHC experiments, as for these the QCD dominated (e.g. multijet) events are a challenging background.

The process where the effects of colour coherence can be studied with the ultimate precision is the $e^{+} e^{-}$annihilation with the centre-of-mass energy close to the mass of the $Z$ resonance. In this process the initial state leaks strongly interacting objects, which eases the calculations of predictions for the final state.

In this analysis we compare the experimental results and predictions based on different models and coherence schemes. We examine experimental measurements based on the OPAL data and the predictions from six models for the coherence as these are implemented in the HERWIG++, Pythia 8 and Vincia Monte Carlo event generators. It was shown before [2,3] that the most favourable events for the studies of colour coherence are the four-jet configurations in which a soft gluon is emitted in the context of a three-jet topology, with two of the three jets approximately collinear. Therefore, to discriminate between the considered models, these events are studied and the recently proposed observables [4], described in details below, are measured.

\section{Observables and theory models}

To reconstruct the hadronic annihilation of the $e^{+} e^{-}$pairs at the $Z$ boson peak (centre-ofmass energy $\sqrt{s}=91.2 \mathrm{GeV}$ ) we apply the Durham jet algorithm [5]. The latter implies inclusive sequential merging of closest clustered objects (jets) $i$ and $j$ using the pair distance measure $y=$ $2 \min \left(E_{i}^{2}, E_{j}^{2}\right)\left(1-\cos \theta_{i j}\right) / s$, with the corresponding energies of the objects (jets) $E_{i}, E_{j}$ and angles $\theta_{i j}$ between them. The studied configurations are characterised with the following quantities: the values of $y$ that correspond to transition from $n+1$ to $n$-jet event configuration, the energies and the angles between the jets.

\subsection{Observables}

Similar to Ref. [4], we select the four jet events in a way that $y^{4 \rightarrow 3}>0.0045$. For these we apply angular cuts $\theta_{12}>2 \pi / 3, \theta_{13}>2 \pi / 3$, and $\theta_{23}<\pi / 6$ and examine $\theta_{14}$, as proposed in 
Ref. [3]; $\theta^{*}=\theta_{24}-\theta_{23}$, as proposed in Ref. [2] and the 2-point energy correlation double ratio $C_{2}^{(\beta)}$, with the $\beta$ parameter equal to $1 / 5$ as proposed in Ref. [6]. In the most general case

$$
C_{2}^{(\beta=1 / 5)}=\frac{\sum_{i} E_{i} \sum_{i<j<k} E_{i} E_{j} E_{k}\left(\theta_{i j} \theta_{i k} \theta_{j k}\right)^{(1 / 5)}}{\left(\sum_{i<j} E_{i} E_{j}\left(\theta_{i j}\right)^{(1 / 5)}\right)^{2}},
$$

but for the nearly collinear (23) jet pair $C_{2}^{(1 / 5)}$ is sensitive to the relative energy of the fourth jet:

$$
C_{2}^{(1 / 5)} \approx \frac{E_{4} \theta_{14}^{1 / 5} \theta_{24}^{1 / 5}\left(E_{1}+E_{2}+E_{3}+E_{4}\right)}{E_{1}\left(E_{2}+E_{3}\right) \theta_{12}^{1 / 5}} \approx \frac{E_{4} \theta_{14}^{1 / 5} \theta_{34}^{1 / 5}\left(E_{1}+E_{2}+E_{3}+E_{4}\right)}{E_{1}\left(E_{2}+E_{3}\right) \theta_{13}^{1 / 5}}
$$

For the selected events with $y^{4 \rightarrow 3}>0.5 y^{3 \rightarrow 2}$ and no angular cuts we examine the $\rho$ variable, proposed in Ref. [3]. The $\rho$ is defined as the ratio of squared jet masses in the end of clustering, when only two jets remain, $\rho=M_{L}^{2} / M_{H}^{2}$ with $M_{H}>M_{L}$.

The sketches for the described variables are shown in Fig. 1.

a)

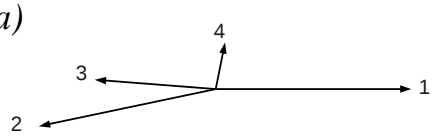

d)

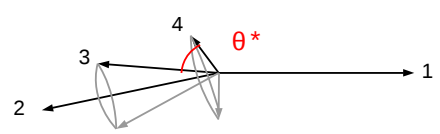

b)

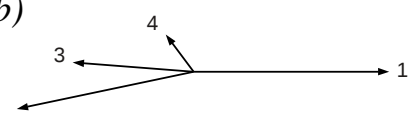

e)

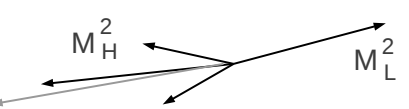

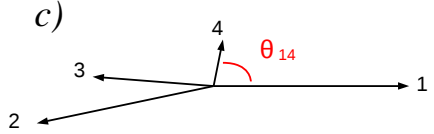

f)

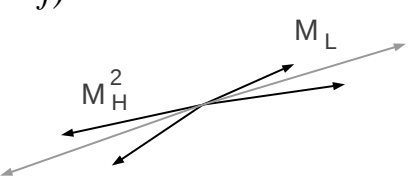

Figure 1: $a$ ), b) The examples of the selected event topologies. c) The observable $\theta_{14}$, the angle between the first and fourth jet in the latter events. $d$ ) The observable $\theta^{*}=\theta_{24}-\theta_{23}$, the difference in opening angles. $e$ ) The event topologies where the third and fourth clusterings occur within the same jet and hence $\rho$ is small. $f$ ) The event topologies with with large $\rho$.

Qualitatively we study the differences between the theoretical models in two ways. First we introduce the asymmetry of a given distribution with respect to an arbitrary dividing point $x_{0}$ :

$$
N_{\text {left }} / N_{\text {right }}=\sum_{x<x_{0}} n(x) / \sum_{x>x_{0}} n(x),
$$

where $n(x)$ is the number of events in histogram bin centred at $x$. The asymmetry is studied for $\theta^{*}$, $C_{2}^{(1 / 5)}$, and $\rho$ with the corresponding dividing points $\theta_{0}^{*}, C_{2,0}^{(1 / 5)}$ and $\rho_{0}$. The second approach is used only for the distribution of $\theta_{14}$. The region of study is divided into three subregions, called towards, central and away. Then, the ratios of number of events in different regions (e.g. $N_{\text {towards }} / N_{\text {away }}$ ) is calculated and compared between data and different Monte Carlo models. The detailed analysis of the asymmetries and the ratios can be found in Ref. [7].

\subsection{Theory models}

The six considered parton shower models are based on the different approaches to parton 
splitting, moment conservation and evolution variables. In the DGLAP approach [8-10], each parton undergoes $1 \rightarrow 2$ splittings and momentum conservation is ensured with a recoil somewhere else in the event. In the approach based on the Catani-Seymour (CS) dipole functions [11] a single parton is produced by a pair of colour connected partons.

The full branching probability in this case includes two cases: when the first parton acts as emitter with the second being the recoiler and vice verse. If the dipole shower uses an evolution with ordering in transverse momentum, the shower correctly reproduces the soft properties of QCD. In the approach of QCD antenna (QA) $[12,13]$ the partons are produced by colour-connected parton pairs with a $2 \rightarrow 3$ splitting, with no distinction between the emitter and recoiler role.

The investigated theory models use the formalisms described above and are implemented in the Herwig++, Pythia 8 and Vincia Monte Carlo generators. Depending on the evolution variables we denote the models as HerwIG $++\tilde{q}^{2}$ (DGLAP), HerWIG $++p_{\perp \text { dip }}^{2}$ (CS), HerwIG ++ $q_{\text {dip }}^{2}(\mathrm{CS}), \mathrm{VINCIA} p_{\perp \text { ant }}^{2}(\mathrm{QA}), \mathrm{VINCIA} m_{\mathrm{ant}}^{2}(\mathrm{QA})$ and PYTHIA $8 p_{\perp \text { evol }}^{2}$ (DGLAP). A more detailed comparison of the model implementations in the context of this analysis can be found in Ref. [7].

To compare the models and their implementation on as equal a footing as possible, the shower and hadronisation parameters have been readjusted using LEP data [4]. This assure the comparison of the models rather than a comparison of the particular MC generator tuning.

\section{Data and MC samples}

The OPAL experiment at the LEP accelerator operated between August 1989 and November 2000. A detailed description of the experiment set-up can be found in Refs. [14-16]. In the first phase of LEP operation, denoted LEP1 (1989 to 1995), the $e^{+} e^{-}$center-of-mass energy was chosen to lie at or near the mass of the $Z$ boson, $\sqrt{s} \approx 91 \mathrm{GeV}$. During the second phase of operation, denoted LEP2 (1995-2000), the center-of-mass energy was increased in successive steps from 130 to $209 \mathrm{GeV}$. Interspersed at various times during the LEP2 operation, calibration runs are collected at the $Z$ boson peak. In this analysis, we utilise a data sample corresponding to an integrated luminosity of $14.7 \mathrm{pb}^{-1}$ collected at $\sqrt{s}=91.2 \mathrm{GeV}$ during the LEP2 calibration runs. This allows us to exploit conditions when the detector was operating in its final, most advanced configuration. This sample is of sufficient size such that systematic uncertainties dominate the statistical terms. To correct the data in order to account for experimental acceptance and efficiency, simulated event samples produced with MC event generators are used. The process $e^{+} e^{-} \rightarrow q \bar{q}$ is simulated using Pythia 6.1 [17] at $\sqrt{s}=91.2 \mathrm{GeV}$. Corresponding samples using HeRWIG $6.2[18,19]$ are used for systematic checks. We examine the MC events at two levels. We refer to "hadron level" as events without event selection, and without simulation of the detector acceptance and resolution, for which all particles with lifetimes less than 300 ps decay. In contrast, "detector level" refers to MC events that are processed through the simulation of the OPAL detector and have been reconstructed using the same software procedures that are applied to the data.

\section{Selection of events, reconstruction and correction}

The selection of hadronic annihilation events is the same as described in Ref. [20]. The events are reconstructed using the calorimeter clusters and the charged tracks (a minimum of five tracks 
is required). The applied selection of charged tracks and electromagnetic clusters is described in Ref. [21]. Basically, charged tracks are required to have transverse momentum relative to the beam axis larger than $0.15 \mathrm{GeV}$, and photons to have energies larger than $0.10 \mathrm{GeV}(0.25 \mathrm{GeV})$ in the barrel (endcap) region of the electromagnetic calorimeter. To avoid double-counting of energy between tracks and electromagnetic clusters, an energy-flow algorithm [24,25] is applied, which matches the tracks and clusters and retains only those clusters that are not associated with a track. The obtained set of tracks and clusters was used to calculate the thrust axis in the event. Then, a $\left|\cos \theta_{\mathrm{T}}\right|<0.90$ condition is applied, where $\theta_{\mathrm{T}}$ is the polar angle of the thrust axis [22,23] with respect to the beam axis. In this way a total of 397452 candidate hadronic annihilation events are selected, with a negligible expected background.

For the MC detector-level samples used to correct the data in addition to the described selection procedure, the radiative corrections are applied by requiring $\sqrt{s}-\sqrt{s^{\prime}}<1 \mathrm{GeV}$, where $\sqrt{s^{\prime}}$ is the effective center-of-mass energy after initial-state radiation.
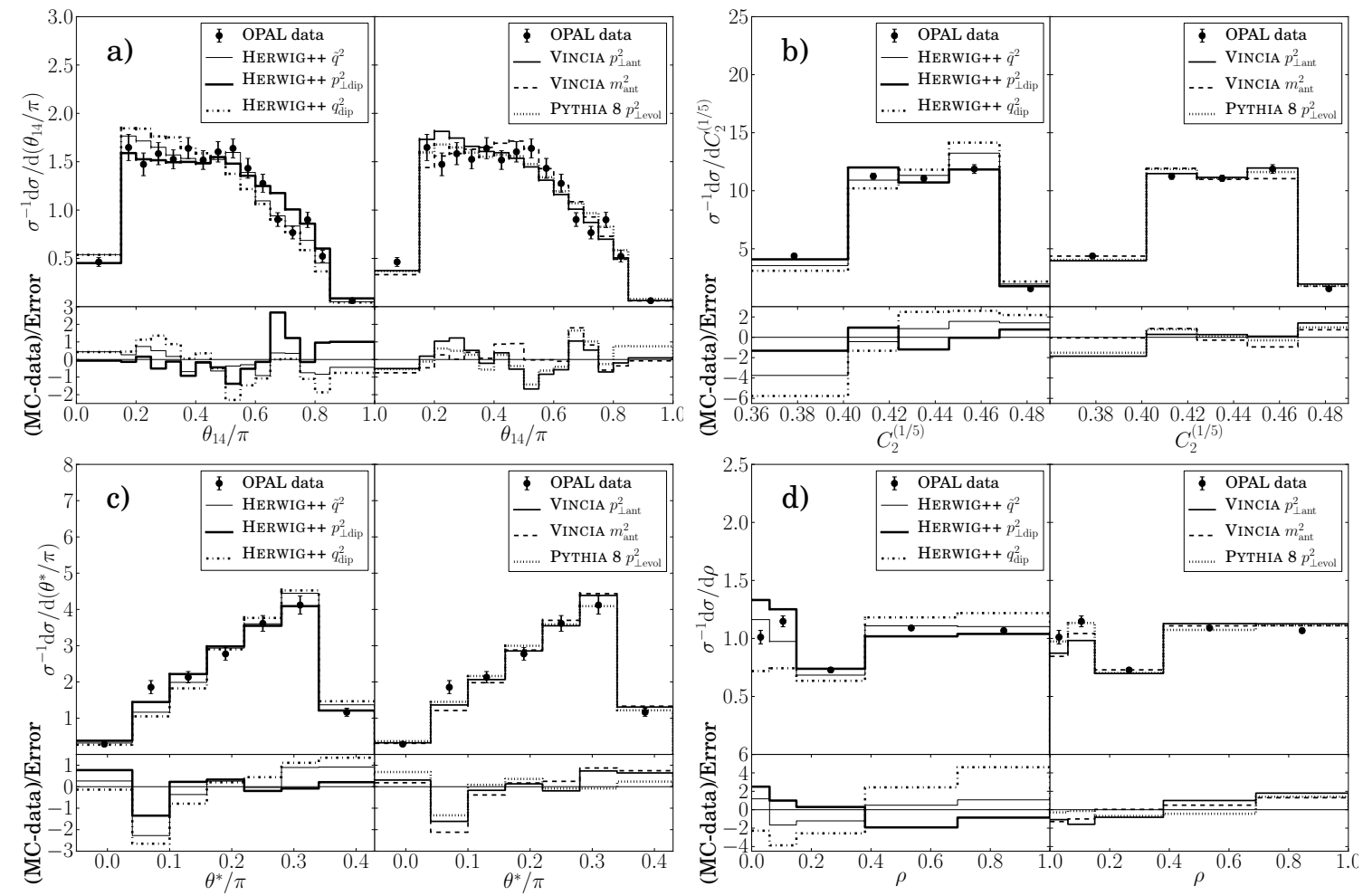

Figure 2: The corrected distributions of a) $\theta_{14} / \pi$, b) $C_{2}^{(1 / 5)}$, c) $\theta^{*} / \pi$ and d) $\rho$ in comparison with the predictions of HERWIG++, PYTHIA 8 and VinCIA.

The correction of the obtained distributions for the detector effects is done with unfolding procedure using the MC samples described in Sec. 3. The corrected distributions are presented in Fig. 2 together with the predictions of the models described in Sec. 2.2. To examine the quality of the predictions a bin-by-bin $\chi^{2}$ is calculated for every prediction as well as the corresponding $p$-value, obtained from $\chi^{2}$ and number degrees of freedom $n_{\text {dof }}$. The results are presented in Tab. 1 . 


\begin{tabular}{lrrrrrr}
\hline & HERWIG++ $\tilde{q}^{2}$ & HERWIG++ $p_{\perp \text { dip }}^{2}$ & HERWIG++ $q_{\text {dip }}^{2}$ & PYTHIA $8 p_{\perp \text { evol }}^{2}$ & VINCIA $p_{\perp \text { ant }}^{2}$ & VINCIA $m_{\text {ant }}^{2}$ \\
\hline$\theta_{14} / \pi$ & $4.8 / 15(99.3 \%)$ & $12.8 / 15(61.8 \%)$ & $16.4 / 15(35.6 \%)$ & $8.3 / 15(91.1 \%)$ & $8.1 / 15(92.0 \%)$ & $7.1 / 15(95.5 \%)$ \\
$\theta^{*} / \pi$ & $6.1 / 6(41.2 \%)$ & $2.8 / 6(83.3 \%)$ & $8.8 / 6(18.5 \%)$ & $2.5 / 6(86.8 \%)$ & $3.3 / 6(77.0 \%)$ & $5.1 / 6(53.1 \%)$ \\
$C_{2}^{(1 / 5)}$ & $16.2 / 4(0.0 \%)$ & $6.0 / 4(19.9 \%)$ & $41.2 / 4(0.0 \%)$ & $4.3 / 4(36.7 \%)$ & $5.3 / 4(25.8 \%)$ & $2.3 / 4(68.1 \%)$ \\
$\rho^{2}$ & $6.6 / 4(15.9 \%)$ & $10.1 / 4(3.9 \%)$ & $42.8 / 4(0.0 \%)$ & $2.6 / 4(62.7 \%)$ & $6.8 / 4(14.7 \%)$ & $3.9 / 4(42.0 \%)$ \\
\hline
\end{tabular}

Table 1: The $\chi^{2} / n_{\text {dof }}$ values (with $p$-values in parentheses) for the considered observables.

\section{Summary and conclusion}

The data, corresponding to a sample of about 397000 hadronic annihilation events, collected with the OPAL detector was analysed.

The observables that are sensitive to QCD colour coherence, the ordering parameter in parton showers, and to whether four-jet events arise from two separate $1 \rightarrow 2$ splittings or from a $1 \rightarrow 3$ splitting were considered in the data and compared to the predictions for the different parton shower models as implemented in the Herwig++, Pythia 8, and Vincia Monte Carlo event generators.

Each of the six investigated models is found to be in reasonable agreement with the data. Among them the Pythia 8 and Vincia models with evolution in transverse momentum give the best description of the measurements presented here. The variant of HERWIG++ with a $q_{\mathrm{dip}}^{2}$-ordered dipole shower is found to provide the least satisfactory description of the data. This model does not contain coherence; it has intentionally been introduced to confront it with coherent evolution.

Important to admit this analysis as the first one that allows to distinguish between the predictions of PyThia 8 and Vincia, or between the different variants of Vincia. For instance, the study of the asymmetry of the ratio of squared jet masses, shows that VINCIA predicts somewhat too many opposite-side events (i.e., events with two $1 \rightarrow 2$ splittings) compared to same-side events (i.e., events with a $1 \rightarrow 3$ splitting), and that the data prefer Pythia 8.

Our results emphasise the importance of incorporating coherence into the description of the QCD multijet process.

\section{Acknowledgements}

We would like to express our gratitude to S. Bentvelssen, S. Bethke, J.W. Gary and K. Rabbertz for their careful review of the analysis.

\section{References}

[1] See the author list of the OPAL collaboration in G. Abbiendi et al., Eur. Phys. J. C 71 (2011) 1733, [arXiv:1101.1470].

[2] S. Plätzer and S. Gieseke, JHEP 1101 (2011) 024, [arXiv: 0909.5593 ].

[3] J.A. Maestre et al., [arXiv:1203.6803].

[4] N. Fischer, S. Gieseke, S. Plätzer and P.Z. Skands, Eur. Phys. J. C 74 (2014) 2831, [arXiv:1402.3186].

[5] S. Catani, Y.L. Dokshitzer, M. Olsson, G. Turnock and B. Webber, Phys. Lett. B 269 (1991) 432. 
[6] A.J. Larkoski, G.P. Salam and J. Thaler, JHEP 1306 (2013) 108, [arXiv: 1305.0007 ] .

[7] OPAL Coll., N. Fischer et al., arXiv:1505.01636.

[8] V.N. Gribov and L.N. Lipatov, Sov. J. Nucl. Phys. 15 (1972) 438.

[9] G. Altarelli and G. Parisi, Nucl. Phys. B 126 (1977) 298.

[10] Y.L. Dokshitzer, Sov. Phys. JETP 46 (1977) 641.

[11] S. Catani and M.H. Seymour, Nucl. Phys. B 485 (1997) 291, [arXiv: hep-ph/9605323] .

[12] G. Gustafson and U. Pettersson, Nucl. Phys. B 306 (1988) 746.

[13] D.A. Kosower, Phys. Rev. D 71 (2005) 045016, [arXiv: hep-ph/0311272].

[14] OPAL Coll., K. Ahmet et al., Nucl. Instrum. Meth. A 305 (1991) 275.

[15] OPAL Coll., S. Anderson et al., Nucl. Instrum. Meth. A 403 (1998) 326.

[16] G. Aguillion et al., Nucl. Instrum. Meth. A 417 (1998) 266.

[17] T. Sjöstrand et al., Comput. Phys. Commun. 135 (2001) 238, [arXiv: hep-ph/0010017]

[18] G. Marchesini et al., Comput. Phys. Commun. 67 (1992) 465.

[19] G. Corcella et al., JHEP 0101 (2001) 010, [arXiv: hep-ph/0011363 ].

[20] OPAL Coll., G. Alexander et al., Z. Phys. C 52 (1991) 175.

[21] OPAL Coll., G. Abbiendi et al., Eur. Phys. J. C 40 (2005) 287, [arXiv: hep-ex/ 0503051 ] .

[22] S. Brandt, C. Peyrou, R. Sosnowski and A. Wroblewski, Phys. Lett. 12 (1964) 57.

[23] E. Farhi, Phys. Rev. Lett. 39 (1977) 1587.

[24] OPAL Coll., K. Ackerstaff et al., Eur. Phys. J. C 2 (1998) 213, [arXiv: hep-ex/9708018] .

[25] OPAL Coll., G. Abbiendi et al., Eur. Phys. J. C 12 (2000) 567, [arXiv: hep-ex/9908002] . 